Audiology

Neurotology
Audiol Neurotol 2010;15:291-302

DOI: $\underline{10.1159 / 000283006}$
Received: August 7, 2009

Accepted after revision: November 27, 2009

Published online: February 11, 2010

\title{
Round Window Membrane Implantation with an Active Middle Ear Implant: A Study of the Effects on the Performance of Round Window Exposure and Transducer Tip Diameter in Human Cadaveric Temporal Bones
}

\author{
Stéphane Tringali ${ }^{a-d}$ Kanthaiah Koka ${ }^{a} \quad$ Arnaud Deveze $^{a}$, b, e N. Julian Holland ${ }^{a}$ \\ Herman A. Jenkins ${ }^{b}$ Daniel J. Tollin ${ }^{a, b}$ \\ Departments of a Physiology and Biophysics and ${ }^{b}$ Otolaryngology, University of Colorado Medical School, \\ Aurora, Colo., USA; CUMR5020 Neurosciences Sensorielles, Comportement, Cognition, CNRS, Université de Lyon, \\ Université Claude-Bernard Lyon-1, Lyon, 'Department of Otology and Otoneurosurgery, Centre Hospitalier \\ Lyon-Sud, Hospices Civils de Lyon, Pierre-Bénite, and ' Department of Otology and Otoneurosurgery, CHU Nord, \\ Assistance Publique, Hôpitaux de Marseille, Université de La Méditerranée, Marseille, France
}

\section{Key Words}

Round window $\cdot$ Active middle ear implant $\cdot$ Laser Doppler velocimetry $\cdot$ Temporal bone

\footnotetext{
Abstract

Objectives: To assess the importance of 2 variables, transducer tip diameter and resection of the round window (RW) niche, affecting the optimization of the mechanical stimulation of the RW membrane with an active middle ear implant (AMEI). Materials and Methods: Ten temporal bones were prepared with combined atticotomy and facial recess approach to expose the RW. An AMEl stimulated the RW with 2 ball tip diameters $(0.5$ and $1.0 \mathrm{~mm}$ ) before and after the resection of the bony rim of the RW niche. The RW drive performance, assessed by stapes velocities using laser Doppler velocimetry, was analyzed in 3 frequency ranges: low $(0.25$ $1 \mathrm{kHz})$, medium (1-3 kHz) and high (3-8 kHz). Results: Driving the RW produced mean peak stapes velocities $\left(H_{E V}\right)$ of 0.305 and $0.255 \mathrm{~mm} / \mathrm{s} / \mathrm{V}$ at $3.03 \mathrm{kHz}$, respectively, for the
}

1- and 0.5-mm tips, with the RW niche intact. Niche drilling increased the $H_{E V}$ to 0.73 and $0.832 \mathrm{~mm} / \mathrm{s} / \mathrm{V}$ for the 1 - and $0.5-\mathrm{mm}$ tips, respectively. The tip diameter produced no difference in output at low and medium frequencies; however, the $0.5-\mathrm{mm}$ tip was 5 and $6 \mathrm{~dB}$ better than the $1-\mathrm{mm}$ tip at high frequencies before and after niche drilling, respectively. Drilling the niche significantly improved the output by $4 \mathrm{~dB}$ at high frequencies for the 1-mm tip, and by 6 and 10 $\mathrm{dB}$ in the medium- and high-frequency ranges for the 0.5$\mathrm{mm}$ tip. Conclusion: The AMEl was able to successfully drive the RW membrane in cadaveric temporal bones using a classical facial recess approach. Stimulation of the RW membrane with an AMEl without drilling the niche is sufficient for successful hearing outputs. However, the resection of the bony rim of the RW niche significantly improved the RW stimulation at medium and higher frequencies. Drilling the niche enhances the exposure of the RW membrane and facilitates positioning the implant tip.

Copyright $\odot 2010$ S. Karger AG, Basel 


\section{Introduction}

Conventional hearing aids have advanced tremendously throughout the last decade with miniaturization and improvements in digital signal processing. However, they are subject to limitations such as occlusion of the ear canal, chronic irritation, acoustic feedback, sound distortion and ear level limitations [Kasic and Fredrickson, 2001; Jenkins et al., 2004]. Recently, active middle ear implants (AMEI) have been developed to overcome these problems [Kasic and Fredrickson, 2001; Jenkins et al., 2004, 2007, 2008]. The floating mass transducer of the Vibrant Soundbridge from Med-El (Innsbruck, Austria), the Middle Ear Transducer ${ }^{\mathrm{TM}}$ from Otologics LLC (Boulder, Colo., USA) and the Esteem 2 (Envoy Medical, St. Paul, Minn., USA) are three commercially available AMEI for the treatment of moderate-to-severe sensorineural hearing loss in adults [Kasic and Fredrickson, 2001; Sterkers et al., 2003; Jenkins et al., 2004, 2007, 2008; Barbara et al., 2009]. Some of these devices have been applied in conductive or mixed hearing loss therapy [Colletti et al., 2006; Siegert et al., 2007; Venail et al., 2007; Hüttenbrink et al., 2008; Tringali et al., 2008, 2009; Beltrame et al., 2009; Lefebvre et al., 2009; Martin et al., 2009] but none are currently FDA approved for this application. In these cases, the AMEI transducer tips have been modified so that they can be attached to various parts of the ossicular chain, such as the capitulum or footplate of the stapes. However, in some clinical situations, the remaining ossicles are not available (e.g. after multiple surgeries for cholesteatoma and stapedectomy or for chronic otitis media). For these conditions, providing the mechanical drive via the AMEI through the round window (RW) membrane is a viable option to deliver stimulation to the inner ear. Recently, the authors have reported initial clinical results with direct sound transfer to the inner ear through the RW membrane using the Otologics AMEI [Lefebvre et al., 2009; Martin et al., 2009; Tringali et al., 2009]. The feasibility of acoustic transfer to the inner ear via the RW was initially reported in humans using the floating mass transducer [Colletti et al., 2006].

Currently, there is limited knowledge regarding the relative importance of AMEI placement factors that may influence the efficacy of mechanically stimulating the RW membrane with an AMEI. For example, variance in the positioning of the implant with regard to the RW membrane may be an important variable. There is no consensus in the literature regarding the optimal site for the positioning, or effective methods of mechanically coupling, the AMEI to the RW for optimal stimulation. The aims of this study were to assess in human cadaveric temporal bones the effects on performance of two RW AMEI placement variables: the diameter of the spherical tip of the transducer and widening the exposure of the RW membrane.

\section{Material and Methods}

\section{Temporal Bone Preparation}

The use of temporal bone tissue was in compliance with the University of Colorado Denver Institutional Biosafety Committee. Ten temporal bones were evaluated. All temporal bones were obtained from cadavers that were undergoing autopsy with permission to use tissues and organs for research, and were frozen prior to use (LifeLegacy Foundation, Tucson, Ariz., USA). The temporal bones were prepared using a Schuknecht plug cutter [Nadol, 1996]. These bones were from individuals with no history of middle ear disease (except presbyacusis). In each temporal bone, after thawing, the tympanic membrane and middle ear were inspected with an operating microscope (Zeiss, Oakland, Calif., USA) to make sure the ear appeared normal; bones with abnormal tympanic membranes or middle ear diseases (anomaly or fixation of the ossicular chain) were not used.

For each temporal bone, a canal-wall-up mastoidectomy was performed, and the facial recess opened to visualize the RW and stapes. The stapedius tendon was severed in order to maximize the exposure of the stapes. In all cases, the mastoid segment of the facial nerve and the chorda tympani were preserved in order to provide the same view of the stapes footplate, posterior crus and RW as that obtainable in surgical exposures in patients [Lefebvre et al., 2009; Tringali et al., 2009]. The RW was inspected and the false membrane removed. The mechanical properties of the middle and inner ear were first confirmed by measuring the stapes and RW membrane velocities with acoustic stimulation from the external auditory canal via an appropriately calibrated insert earphone. The bones with acoustic stapes-velocity transfer functions outside the range described in the criteria by Rosowski et al. [2007] were excluded. In all cases, the bony overhang of the RW niche was initially preserved. The prepared bones were firmly mounted in a holding block for study and were kept hydrated with saline solution $(0.9 \%)$ to prevent any change in the transfer function [Willi et al., 2002].

\section{Acoustic Calibration and Stimulus Presentation}

All experiments were performed in a double-walled, soundattenuating room (Industrial Acoustics Company, Bronx, N.Y., USA). Acoustic stimuli were presented via a foam insert earphone fixed into the bony ear canal stabilized with super glue. Vaseline was added as needed to the perimeter of the foam to prevent acoustic leakage. The earphone (Tucker Davis Technologies model CF1, TDT, Alachua, Fla., USA) was driven by sinusoidal stimuli generated by a TDT RX6 digital-to-analog converter. A microphone (Brüel and Kjær Type 4182, Norcross, Ga., USA) with a precalibrated $50-\mathrm{mm}$-long probe tube attached was positioned to within $1 \mathrm{~mm}$ of the center of the tympanic membrane by drilling a small hole in the posterior part of the bony ear canal. The mi- 


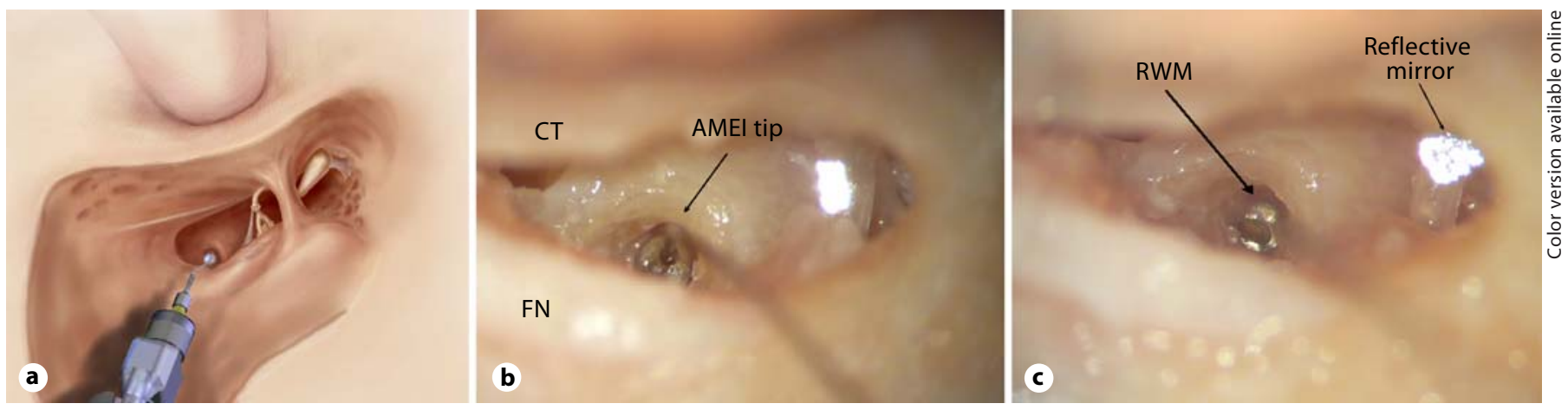

Fig. 1. a Schematic diagram explaining the position of the tip of the transducer for RW application in a left ear (schematic diagram provided by Otologics LLC). b 1.0-mm-diameter AMEI tip after final positioning onto the RW niche in a left ear. Note that the RW membrane itself is fully hidden by the AMEI ball tip, precluding visual confirmation that the AMEI tip is in contact with the mem- brane. $\mathrm{CT}=$ Chorda tympani; $\mathrm{FN}$ = facial nerve. c 0.5 - $\mathrm{mm}$-diameter AMEI tip after final positioning onto the RW niche in a left ear. With the $0.5-\mathrm{mm}$ ball tip, some part of the RW membrane (RWM) remains visible, allowing confirmation of the direct placement of the tip onto the membrane. The reflective mirror is on the posterior crus of the stapes. crophone output was preamplified (Brüel and Kjær Dual Microphone Supply 5935L) and recorded using the analog-to-digital converters of a TDT RX6. The sealed earphone assembly was calibrated for tones from $100 \mathrm{~Hz}$ to $14 \mathrm{kHz}$ by the microphone. The calibration data were used to compute digital filters that equalized the system and yielded flat responses $( \pm 2 \mathrm{~dB})$ up to $14 \mathrm{kHz}$. After equalization, the maximum outputs across the frequencies of interest were at least $100 \mathrm{~dB}$ SPL. The stimuli were presented either through the acoustic closed-field earphones as above or via an AMEI transducer placed on the RW membrane. The stimuli were $30 \mathrm{log}$-spaced frequencies $(0.25-14 \mathrm{kHz} ; 5$ frequencies/octave) and in 5-dB steps spanning 80-100 dB SPL. The stimuli were generated in MATLAB (version 7.1; The MathWorks Inc., Natick, Mass., USA) and presented at a nominal sampling rate of $100 \mathrm{kHz}$ at full 24-bit resolution using the TDT system III hardware (TDT RX6). Stimulus intensity was controlled via a TDT PA5 attenuator. The probe microphone signals near the tympanic membrane, and the stapes velocity output from the laser Doppler vibrometer (LDV) were simultaneously captured at $100 \mathrm{kHz}$ via the analogto-digital converters on a TDT RP2.1 and stored to disk.

\section{Measurements of Stapes Velocity}

Microsphere (50- $\mu \mathrm{m}$-diameter beads) reflective mirrors $(0.5$ $\mathrm{mm}^{2}$; Scotchlite ${ }^{\mathrm{TM}}$; $3 \mathrm{M}$, St. Paul, Minn., USA) were placed on the stapes capitulum just on top of the insertion of the stapedius tendon and on the RW membrane to improve the LDV signal (Model No. HLV-1000 with CLV-700 head; Polytec Inc., Waldbronn, Germany). The LDV controller sensitivity was typically set to 25 $\mathrm{mm} / \mathrm{s} / \mathrm{V}$ and the input was low-pass- and high-pass-filtered at 30 $\mathrm{kHz}$ and $100 \mathrm{~Hz}$, respectively. The LDV beam was positioned via a joystick-controlled aiming prism (HLVMM2; Polytec Inc.); the beam was visualized by a Zeiss microscope, onto which the LDV head was attached. Our bone preparation allowed us to achieve LDV laser angles for stapes velocity measurements between $65^{\circ}$ and $80^{\circ}$ from the axis of piston-like stapes motion. The stapes and RW velocities were first measured with acoustic closed-field stimulation before driving the RW with an AMEI in order to confirm if the stapes and the RW velocities were in the normal range defined by Rosowski et al. [2007]. Stapes velocity was again measured by stimulating the RW with the AMEI. Finally, with the power to the transducer turned off and no stimulus applied, stapes velocity was then remeasured with acoustic stimulation to assess the decrease in stapes velocity due to the transducer pushing against the RW during forward acoustic stimulation.

\section{AMEI Loading on RW and Resection of RW Niche}

The AMEI used in this study was the Otologics Middle Ear Transducer (middle ear transfer, Ossicular Stimulator; Otologics LLC) with 2 ball tip diameters, 0.5 and $1.0 \mathrm{~mm}$. The AMEI was securely fixed to a stereotaxic micromanipulator (Model 1404; David Kopf Instruments, Tujunga, Calif., USA) that was then advanced under microscopic visualization into the niche of the RW (fig. 1a). The first contact of the AMEI with the RW was confirmed by a slight change in the impedance and inductance indicated by the transducer loading assistant (TLA) software and by direct visualization.

The TLA (Otologics Software; Otologics LLC) facilitates the objective assessment of the degree of static loading of the AMEI by measuring and displaying in real time on a PC monitor the electrical impedance magnitude (units of ohms), measured at the resonance frequency of the transducer (determined from the unloaded condition with a resolution of $100 \mathrm{~Hz}$ ), and the inductance (units of millihenry) measured at $1 \mathrm{kHz}$. The TLA is essentially a custom-built LCR (L for inductance, $\mathrm{C}$ for capacitance and R for resistance) meter that measures these values in the same way that a commercial LCR meter does. The measurement of these properties of the AMEI is useful because any electromechanical transducer, such as the AMEI studied here, will exhibit under load an impedance curve with frequency, the shape of which is determined by characteristics of the transducer itself, and by the mass, damping and spring rate of the load. Within a range, the peak impedance at resonance changes nearly linearly with the applied load. The AMEI is connected to the TLA device via a direct connection during the loading phase using an adapter. The TLA was 
Fig. 2. a RW exposure, including the false membrane (arrow), before drilling the niche. With the niche and false membrane intact, the RW membrane is not clearly visible (left ear). b Complete niche drilling, fully exposing the RW membrane.
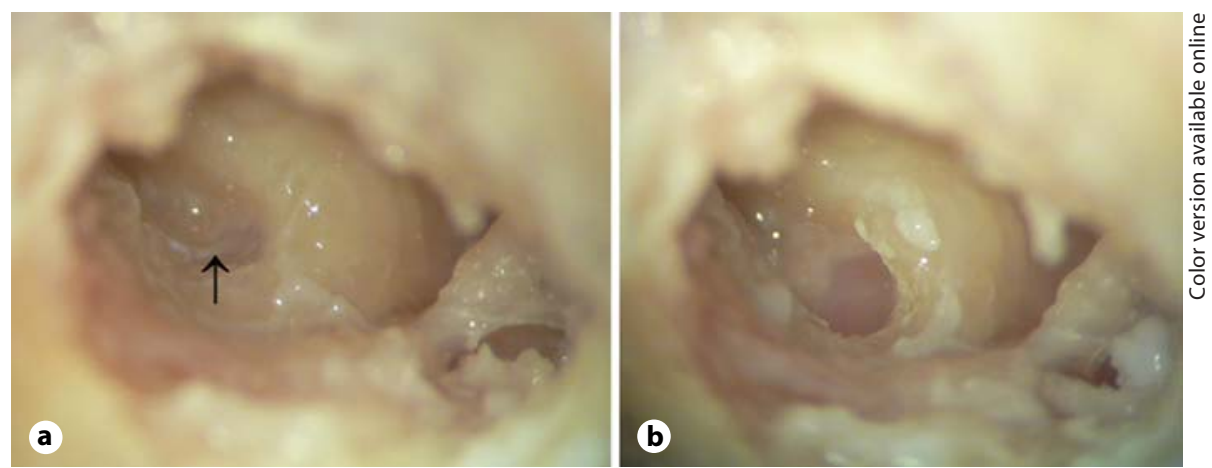

designed to provide information to help surgeons in optimally and consistently loading the transducer onto an ossicle. The TLA software consists of 2 display screens which contain the real-time loading information. The top screen shows the reactive component of the system impedance (similar to the transducer inductance) as measured at $1 \mathrm{kHz}$. The bottom screen shows the magnitude of the peak system electrical impedance, which occurs at the resonance frequency. Finally, the measured DC resistance is displayed as well; according to the manufacturer, this should always be approximately $120 \Omega$ for a properly connected transducer, either loaded or unloaded.

The values from the TLA were used to help load the AMEI tip onto the RW consistently across each experimental condition. For each placement, the AMEI was advanced toward the RW by turning an adjustment screw, where each full turn of the screw advanced the tip by $0.25 \mathrm{~mm}(250 \mu \mathrm{m})$. Using the screw, the AMEI was carefully loaded onto the RW so as to achieve the same change in transducer impedance and inductance (measured by the TLA software) with respect to the unloaded case. Here, the estimated final mechanical force applied to the RW was typically several hundred dynes (approx. 1000 dynes at $1 \mathrm{kHz}$ [Kasic and Fredrickson, 2001]), with the exact value dependent on the individual transducer. The static loading of the AMEI on the RW necessary to maximize performance remains to be determined by future experimental investigation.

Each experiment began by first positioning the 1-mm-diameter ball tip visually inside the niche of the RW, using the micromanipulator and the TLA data (fig. 1b). Afterwards, the stapes velocities were measured. The same measurements were then repeated with the $0.5-\mathrm{mm}$ tip (fig. 1c). After finishing the measurements for the $1-\mathrm{mm}$ and $0.5-\mathrm{mm}$ ball tips, the area caudal to the subiculum (fossula fenestrae cochleae) was reshaped, drilling away the anterior and posterior margins of the bony lip surrounding the RW in order to obtain a complete visualization of the RW membrane (fig. 2). The RW niche was gently drilled in order to obtain a complete visualization of the whole RW annulus without RW membrane damage. In 1 case, the RW membrane was damaged during the drilling, and the temporal bone was excluded. There was no RW membrane damage by the tip of the transducer observed in the present study during the positioning of the transducer. However, RW damage had previously been reported in a clinical report [Martin et al., 2009]. Once the RW niche was drilled, the same procedures described above were repeated on each temporal bone.
The LDV measurement angle to the stapes was kept constant before and after drilling the bony rim of the RW niche. After niche drilling, the AMEI was loaded on the RW at the same angle and with the same range of loads (as assessed by the impedance and inductance measures by the TLA software) as before the niche resection. The AMEI-to-RW angle remained approximately constant as the relative positions of the micromanipulator and temporal bone placement on the stereotaxic apparatus were not changed during the drilling of the bone. This ensured as best as possible the isolation of just the effect on performance of the RW niche. To further isolate the effects of the variables, the data were examined separately within each temporal bone before averaging the performance across bones. At the end of the experiment, a meticulous otoscopic analysis of the middle ear confirmed the integrity of the RW membrane.

Data Processing and Statistical Analysis

Both the closed-field acoustic transfer function $H_{T V}$ and the electrovibrational transfer function $H_{E V}$ were computed from the measured stapes velocities as described by Rosowski et al. [2007] and in the ASTM Standards for Middle Ear Implants [ASTM, 2005]. The closed-field acoustic transfer function $H_{T V}$ was measured as $V_{U} / P_{T}$, where $V_{U}$ is the stapes velocity (root mean square value, rms) for acoustic stimulation and $P_{T}$ is the sound pressure (rms) measured at the tympanic membrane. The closed-field acoustic transfer function with the AMEI loaded (unpowered, no stimulus applied) on the RW, $H_{T V}$ Implanted, was measured as $V_{U}^{\text {Implanted }} / P_{\mathrm{T}}$, where $V_{U}^{\text {Implanted }}$ is the stapes velocity $(\mathrm{rms})$ for acoustic stimulation when the AMEI is placed on the RW, and $P_{T}$ is the sound pressure (rms) measured at the tympanic membrane. The difference in stapes velocity with the AMEI placed passively on the RW $\left(\Delta H_{T V}\right)$ relative to the normal $H_{T V}$ was calculated as $\Delta H_{T V}=20 \cdot \log _{10}\left(H_{T V}\right.$ Implanted $\left./ H_{T V}\right)$. The $\Delta H_{T V}$ estimates via the changes in stapes velocity the acoustic hearing loss that might be expected when the AMEI is placed on the RW. The $\Delta H_{T V}$ was computed for each ball tip size and RW niche conditions.

The electromechanical transfer function $H_{E V}$ was measured as $V_{A} / E$, where $V_{A}$ is the stapes velocity measured in response to mechanical RW stimulation by the AMEI, and $E$ the voltage provided to the AMEI transducer in volts (rms). We used the $H_{E V}$ measurements to objectively compare the relative effects on the AMEI performance of ball tip size and RW niche drilling.

The 2-way repeated ANOVA test was performed to compare the performance of the AMEI with the 2 ball tip diameters and 


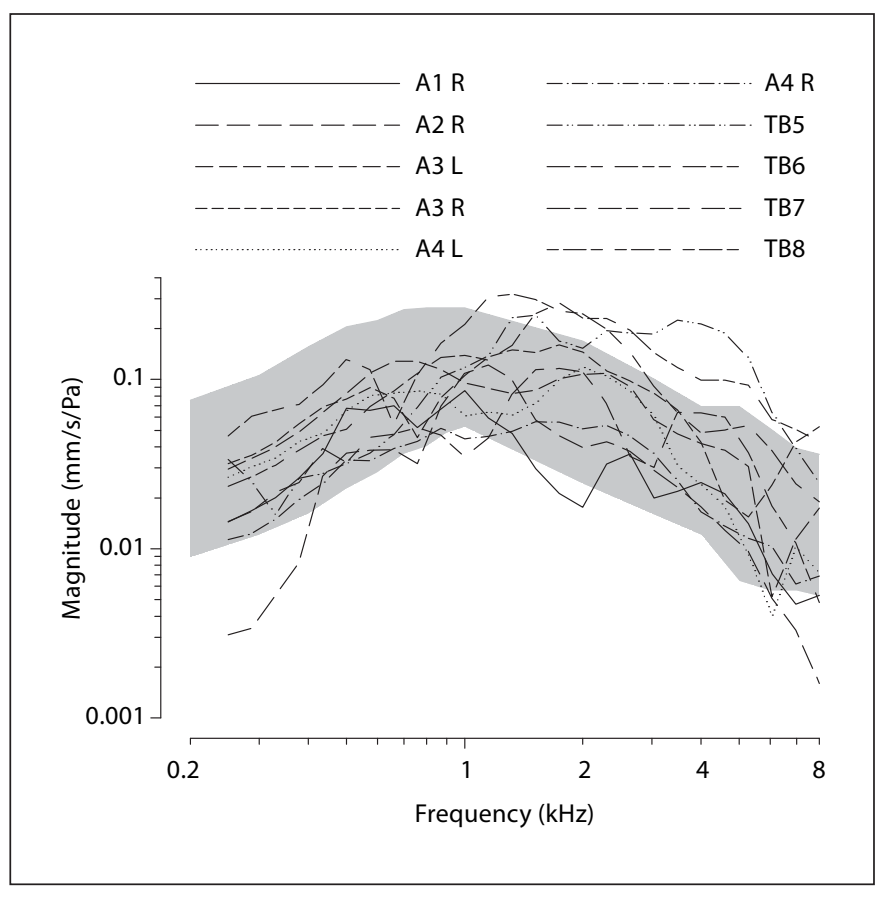

Fig. 3. Closed-field acoustic middle ear transfer functions $\left(H_{T V}\right)$ measured at the stapes in 10 human temporal bones (TB). The shaded area depicts the $95 \%$ CI of the acoustic middle ear transfer functions measured in a large population of temporal bones by Rosowski et al. [2007]. A1-4 = Specimen numbers; $\mathrm{R}=$ right side; $\mathrm{L}=$ left side.

with RW niche drilling. The $\mathrm{p}$ values ( $\mathrm{p}<0.05$ selected as significant value) were shown for each comparison using repeated ANOVA. The data were analyzed and compared in 3 approximately 1-octave-wide frequency ranges: low $(0.25-1 \mathrm{kHz})$, medium $(1-3 \mathrm{kHz})$ and high $(3-8 \mathrm{kHz})$.

\section{Results}

\section{Control Experiment: Expected Variability in}

Performance due to Repeated AMEI Loadings on

the $R W$

In order to explore the effect of the AMEI placement variables on performance, the AMEI was as a necessity placed (i.e. loaded), removed and replaced on the RW membrane of each temporal bone several times. The question naturally arises as to whether the improvement or worsening of performance due to the manipulation of a placement variable in this (or any study) was due to that variable or simply due to the fact that the AMEI was replaced again on the RW. To assess the range of the expected variability in performance of AMEI-driven RW due to the repeated placement of the AMEI, a control experiment was performed in 2 temporal bones where the AMEI was placed, removed and replaced on the RW at least 11 times. In each case, the AMEI was carefully loaded as described above so as to achieve the same change in transducer impedance and inductance (measured by the TLA software) with respect to the unloaded case. The stapes velocity transfer functions $\left(H_{E V}\right)$ were measured for each loading, and the variability in the performance was assessed across the 11 runs. In effect, these control experiments determine the range of the expected performance of RW stimulation in a surgical setting, given a consistent placement of the AMEI on the RW as assessed by visual inspection by the surgeon and an objective loading assessment by changes in transducer impedance and inductance.

These control experiments revealed that repeated loading and unloading of the AMEI on the RW produced quite consistent results, with a range of $H_{E V}$ functions of just $\pm 2.5 \mathrm{~dB}$ when computed across both bones tested and all frequency ranges. Thus, any resulting change in performance that falls in the range of $\pm 2.5 \mathrm{~dB}$ is likely not a meaningful change. In each case, the loading as assessed objectively via the impedance and inductance changes measured by the TLA software (see Material and Methods) was consistent.

\section{Acoustic Closed-Field Transfer Functions $\left(\mathrm{H}_{\mathrm{TV}}\right)$}

The results are from measurements in 10 temporal bones. The closed-field middle ear transfer functions $\left(H_{T V}\right)$ were computed from the recorded stapes velocities. Figure 3 shows the individual measurements of $H_{T V}$ for each temporal bone. The shaded region shows the 95\% confidence interval for $H_{T V}$ from Rosowski et al. [2007]. The data from 4 temporal bones with $H_{T V}$ consistently outside the $95 \%$ confidence interval (CI) were discarded for further studies (these 4 discarded bones are not shown in fig. 3).

\section{Electrovibrational Transfer Function $\left(\mathrm{H}_{\mathrm{EV}}\right)$}

The electrovibrational transfer function $\left(H_{E V}\right)$ measured for the 10 temporal bones with the RW niche intact, along with the mean and 95\% CI computed across bones for the 4 different conditions, are shown in figure 4 . Figure $4 \mathrm{a}$ shows the $H_{E V}$ using a 1-mm ball tip. These transfer functions showed a peak of $0.305 \mathrm{~mm} / \mathrm{s} / \mathrm{V}$ at $3.03 \mathrm{kHz}$. The mean $H_{E V}$ was $0.048,0.15$ and $0.081 \mathrm{~mm} / \mathrm{s} / \mathrm{V}$ for the low-, medium- and high-frequency ranges, respectively. Figure $4 \mathrm{~b}$ shows the $H_{E V}$ using a $0.5-\mathrm{mm}$ ball tip. These transfer functions show a peak of $0.255 \mathrm{~mm} / \mathrm{s} / \mathrm{V}$ at 3.03 


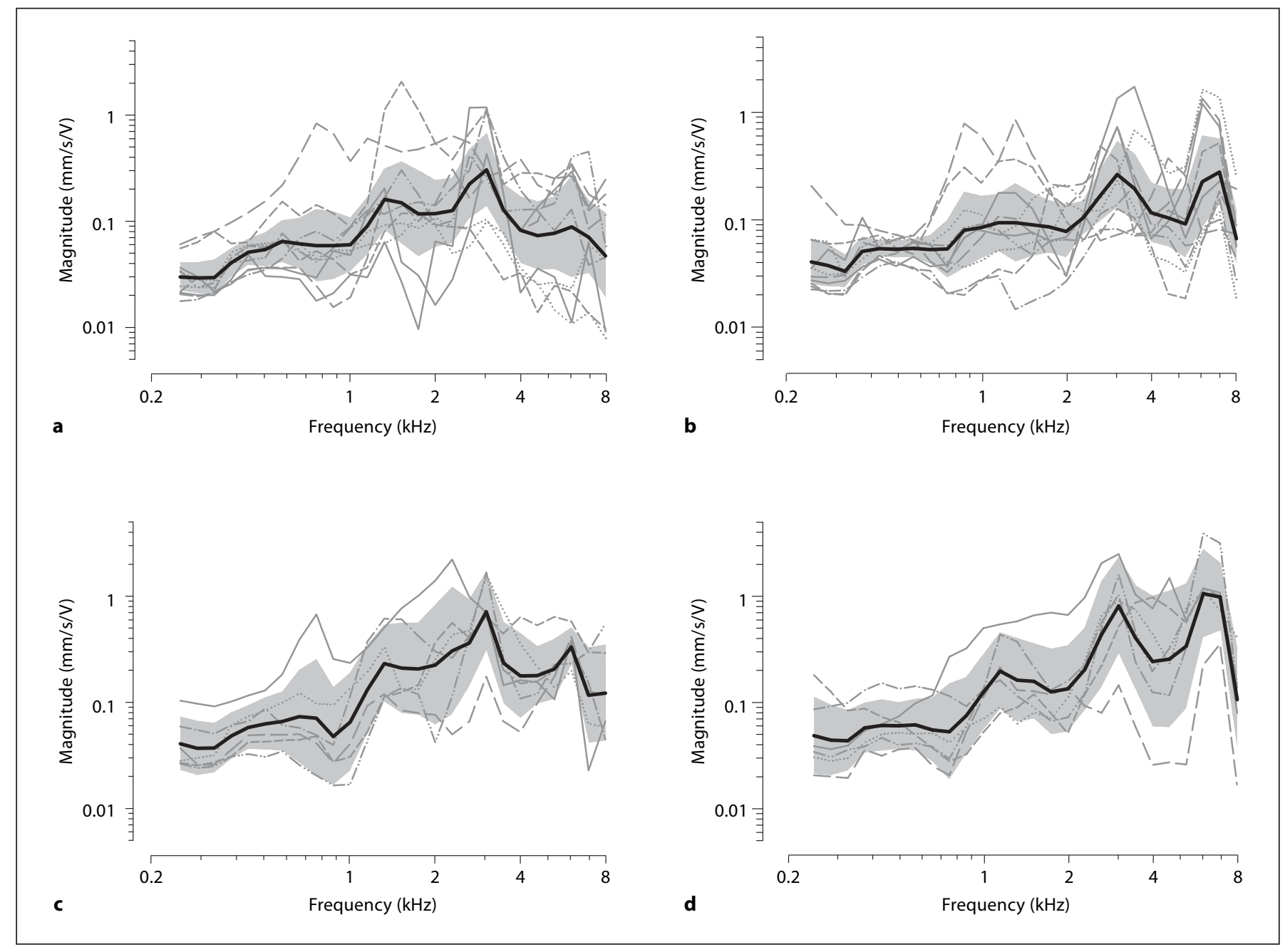

Fig. 4. Electrovibrational transfer functions $\left(H_{E V}\right)$ measured with AMEI on RW. Solid lines and shaded regions in each panel indicate the across-bone means and 95\% CI $(n=10)$, respectively. a $H_{E V}$ for 1-mm ball tip before drilling the RW niche. b $H_{E V}$ for

0.5-mm ball tip before drilling the RW niche. $\mathrm{c} H_{E V}$ for 1-mm ball tip after drilling the RW niche. d $H_{E V}$ for $0.5-\mathrm{mm}$ ball tip after drilling the RW niche.

$\mathrm{kHz}$, and a secondary peak of $0.27 \mathrm{~mm} / \mathrm{s} / \mathrm{V}$ in the $6-$ to 8 -kHz-frequency range. The mean $H_{E V}$ was $0.048,0.113$ and $0.148 \mathrm{~mm} / \mathrm{s} / \mathrm{V}$ for the low-, medium- and high-frequency ranges, respectively.

The $H_{E V}$ measured for the $1-\mathrm{mm}$ and $0.5-\mathrm{mm}$ ball tips after drilling the RW niche are shown in figure $4 \mathrm{c}$ and d, respectively. The $H_{E V}$ increased to $0.73 \mathrm{~mm} / \mathrm{s} / \mathrm{V}$ at 3.03 $\mathrm{kHz}$ for the $1-\mathrm{mm}$ ball tip, and to $0.832 \mathrm{~mm} / \mathrm{s} / \mathrm{V}$ at 3.03 $\mathrm{kHz}$ with a secondary peak of $1.094 \mathrm{~mm} / \mathrm{s} / \mathrm{V}$ in the $6-$ to 8 -kHz-frequency range for the $0.5-\mathrm{mm}$ ball tip. After the RW niche drilled, the mean $H_{E V}$ values were $0.055,0.277$ and $0.198 \mathrm{~mm} / \mathrm{s} / \mathrm{V}$ for the low-, medium- and high-frequency ranges, respectively, for the $1-\mathrm{mm}$ ball tip, and

$0.056,0.266$ and $0.497 \mathrm{~mm} / \mathrm{s} / \mathrm{V}$, respectively, for the same frequency ranges for the $0.5-\mathrm{mm}$ ball tip.

\section{Difference in Acoustic Stapes Velocity with AMEI on $R W\left(\Delta \mathrm{H}_{\mathrm{TV}}\right)$}

The mean difference in acoustic stapes velocity with the AMEI on the RW relative to no AMEI on the RW $\left(\Delta H_{T V}\right)$ computed across the 10 temporal bones along with the 95\% CI for the 4 different conditions are shown in figure 5 . Figure $5 \mathrm{a}$ and $\mathrm{b}$ shows the $\Delta H_{T V}$ using $1-\mathrm{mm}$ and $0.5-\mathrm{mm}$ ball tips, respectively, with the RW niche intact. Figure $5 \mathrm{c}$ and d shows the $\Delta H_{T V}$ measured for 1-mm and $0.5-\mathrm{mm}$ ball tips, respectively, after drilling the RW 

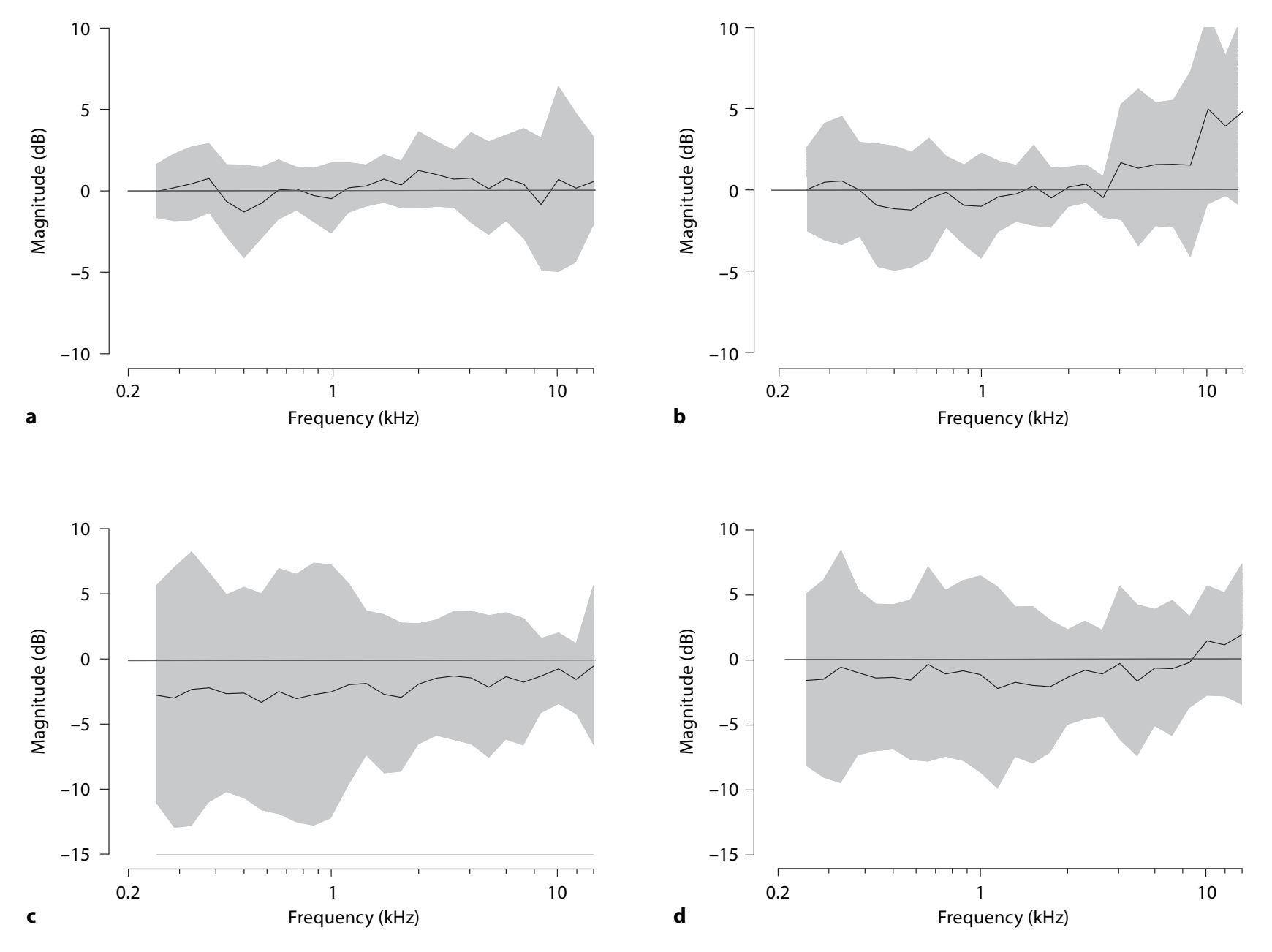

Fig. 5. The difference in acoustic stapes velocity due to the static loading of an AMEI on the RW $\left(\Delta H_{T V}\right)$. Lines and shading show across-bone means and $95 \% \mathrm{CI}$, respectively. a 1-mm ball tip be- fore drilling the RW niche. $\mathbf{b}$ 0.5-mm ball tip before drilling the RW niche. c 1-mm ball tip after drilling the RW niche. d 0.5-mm ball tip after drilling the RW niche. niche (positive values mean improving, and negative values decreasing acoustic stapes velocity). On average, across the frequency ranges tested, the $\Delta H_{T V}$ due to loading the AMEI on the RW was $0.2 \pm 0.6$ and $0.6 \pm 1.7 \mathrm{~dB}$ for the ball tips of $1 \mathrm{~mm}$ and $0.5 \mathrm{~mm}$ diameter, respectively, before RW niche drilling. For the static loads used here (which are surgically relevant), there was a negligible difference in acoustic stapes velocity observed with the different AMEI ball tip diameters before RW niche drilling. After the RW niche was carefully drilled, the $\Delta H_{T V}$ was $-2.1 \pm 0.7$ and $-0.85 \pm 1 \mathrm{~dB}$ for the $1-\mathrm{mm}$ and 0.5 $\mathrm{mm}$ ball tips, respectively. The difference in acoustic stapes velocity was decreased by 2.3 and $0.8 \mathrm{~dB}$, respectively, for the 1-mm and 0.5-mm ball tips by RW niche drilling compared to no drilling. Across all bones and frequencies tested, none of the $\Delta H_{T V}$ reached significance ( $\mathrm{p}>$ $0.05)$.

\section{Effect of Ball Tip Diameter}

To objectively compare the contact effect on performance of the AMEI driving the RW with the 2 different ball tip diameters and with the RW niche intact or drilled, the differences between the $H_{E V}$ functions were computed for each of these conditions to isolate the relevant effects. This $\Delta H_{E V}$, defined here by $20 \cdot \log _{10}$ $\left(H_{E V}{ }^{1-m m ~ t i p} / H_{E V}^{0.5-m m ~ t i p}\right)$, was computed for each bone 


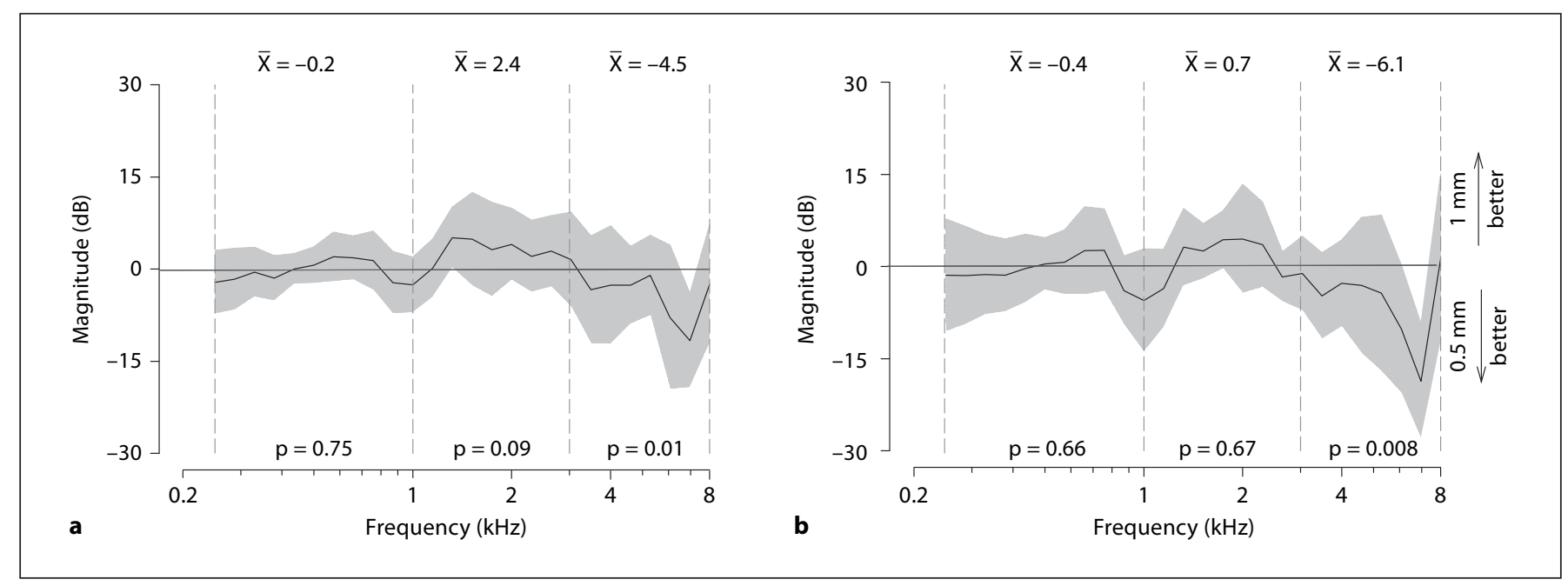

Fig. 6. Effect of ball tip diameter on performance with the AMEI on the RW measured by the difference between the $H_{E V}$ functions in figure 4 . Positive $\Delta H_{E V}$ indicate that the $1.0-\mathrm{mm}$ ball tip outperforms the $0.5-\mathrm{mm}$ tip. Negative $\Delta H_{E V}$ indicate the $0.5-\mathrm{mm}$ tip outperforms the 1.0-mm tip. Solid lines and shading indicate the across-bone mean $\Delta H_{E V}$ and $95 \% \mathrm{CI}$, respectively. The mean
$\Delta H_{E V}$ computed for the 3 frequency ranges is reported at the top of each panel. The resulting $\mathrm{p}$ values of the ANOVA performed on the $\Delta H_{E V}$ are reported at the bottom. a $\Delta H_{E V}$ between $1-\mathrm{mm}$ and $0.5-\mathrm{mm}$ ball tips before drilling the RW niche. $\mathbf{b} \Delta H_{E V}$ between $1-\mathrm{mm}$ and $0.5-\mathrm{mm}$ ball tips after drilling the RW niche. individually, and then these differences were averaged across the 10 bones. This procedure was done to isolate the effects on performance of the variable being studied (e.g. tip size) from inter-bone differences in $H_{E V}$. In this section, the effect of the ball tip diameter was examined by computing the $\Delta H_{E V}$ produced by the 1-mm and the $0.5-\mathrm{mm}$ ball tips under the $2 \mathrm{RW}$ niche conditions, intact or drilled.

Figure 6a shows the $\Delta H_{E V}$ resulting from the 2 tip diameters before drilling the RW niche. In both parts of figure 6 , negative values indicate that the $0.5-\mathrm{mm}$ ball tip produced a better performance (i.e. larger $H_{E V}$ ), while positive values indicate that the $1-\mathrm{mm}$ tip produced a better performance. With the RW niche intact, the ANOVA indicated no significant effect of the ball tip diameter on performance $[\mathrm{F}(1,9)=0.1 ; \mathrm{p}=0.75 ; \mathrm{n}=10]$ in the lowfrequency range, with a mean difference of only $-0.2 \mathrm{~dB}$. No significant differences were found either in the mid-frequency range, with a mean difference of $+2.4 \mathrm{~dB}$ $[\mathrm{F}(1,8)=2.93 ; \mathrm{p}=0.09 ; \mathrm{n}=10]$. In the high-frequency range, however, there was a significant effect of the ball tip diameter $[\mathrm{F}(1,6)=6.44 ; \mathrm{p}=0.012 ; \mathrm{n}=10]$ with a mean difference of $-4.5 \mathrm{~dB}$. In the last case, the negative difference indicates that the $0.5-\mathrm{mm}$ tip significantly outperforms the $1.0-\mathrm{mm}$ tip by $4.5 \mathrm{~dB}$. This value $(4.5 \mathrm{~dB})$ also exceeds the range of expected variability due to repeat loadings of the AMEI on the RW (approx. $\pm 2.5 \mathrm{~dB}$ ) de- tailed in the control experiments (see subsection Control Experiment above).

Figure $6 \mathrm{~b}$ shows the comparison between the $H_{E V}$ of $1-\mathrm{mm}$ and $0.5-\mathrm{mm}$ ball tips after drilling the RW niche. There was no significant effect of the ball tip diameter on performance $[F(1,9)=0.19 ; \mathrm{p}=0.66 ; \mathrm{n}=6]$ in the lowfrequency range with a mean difference of $-0.36 \mathrm{~dB}$. There was also no significant difference $[\mathrm{F}(1,8)=0.17$; $\mathrm{p}=0.67 ; \mathrm{n}=6$ ] between ball tip diameters in the midfrequency range with a mean difference of $0.67 \mathrm{~dB}$. As before, however, there was a significant effect on performance of the ball tip diameter $[\mathrm{F}(1,6)=7.39 ; \mathrm{p}=0.008$; $\mathrm{n}=6]$ in the high-frequency range, with a mean difference of $-6.1 \mathrm{~dB}$. In the last case, the negative difference indicates that the $0.5-\mathrm{mm}$ ball tip significantly outperformed the $1.0-\mathrm{mm}$ tip by $6.1 \mathrm{~dB}$ in only the high-frequency range (see also subsection Control Experiment above).

\section{Effect of Drilling RW Niche}

As in the subsection Effect of Ball Tip Diameter above, the differences in performance in terms of $\Delta H_{E V}=$ $20 \cdot \log _{10}\left(H_{E V}\right.$ With niche drilled $/ H_{E V}$ With intact niche $)$ were computed for the 2 ball tip diameters before and after drilling the niche. Figure $7 \mathrm{a}$ and $\mathrm{b}$ shows the performance differences resulting from drilling the RW niche in terms of $\Delta H_{E V}$ for the 1-mm and 0.5-mm tips, respectively. Here, 


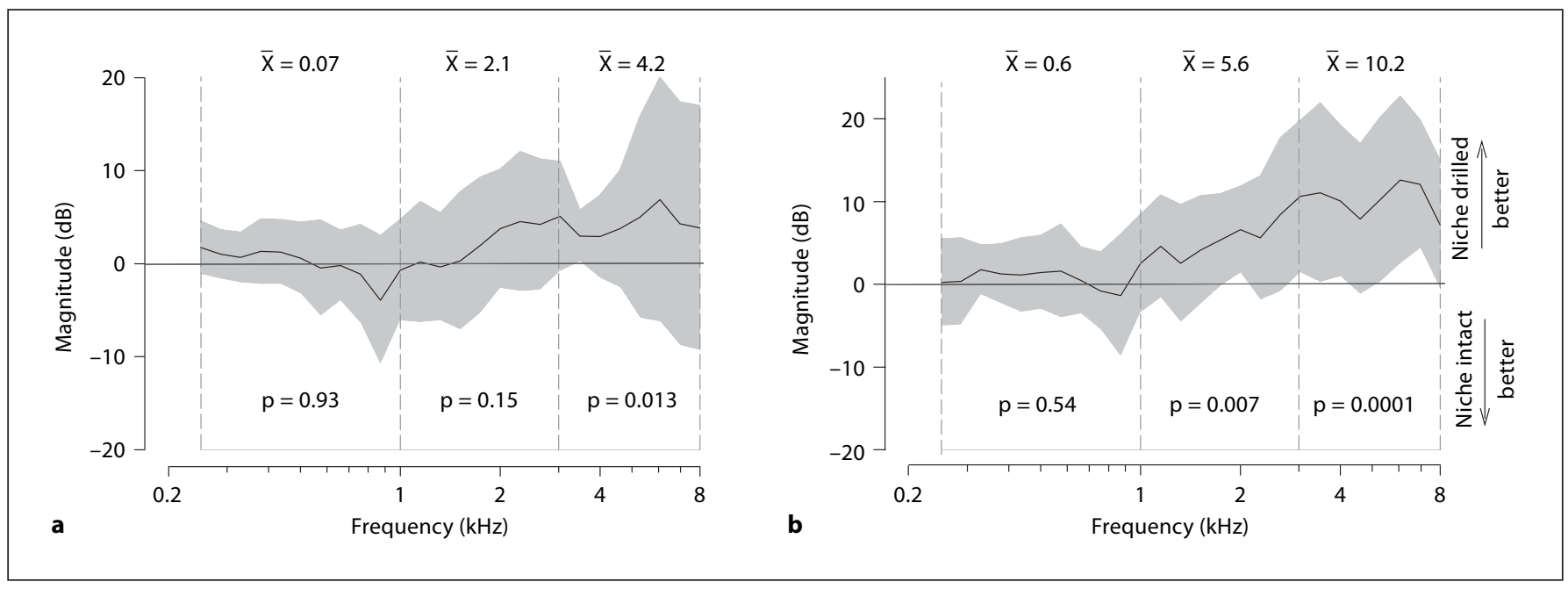

Fig. 7. Effect of RW niche drilling on performance with the AMEI placed on the RW measured in $\Delta H_{E V}$. Positive $\Delta H_{E V}$ indicate that niche drilling outperforms the intact niche condition. Negative
$\Delta H_{E V}$ indicate that the intact niche outperforms the drilled niche. a 1-mm ball tip with and without drilling the RW niche. b $0.5-\mathrm{mm}$ ball tip with and without drilling the RW niche. positive values indicate that RW niche drilling improved performance, while negative values indicate that the RW intact condition produced the best results.

Figure 7a shows the $\Delta H_{E V}$ for the 1-mm ball tip with the RW intact and after drilling. There was no significant effect on performance of RW niche drilling $[\mathrm{F}(1,9)=0.01$; $\mathrm{p}=0.93 ; \mathrm{n}=6]$ in the low-frequency range, with a mean of $0.07 \mathrm{~dB}$. There was also no significant difference $[\mathrm{F}(1$, $8)=2.12 ; p=0.148 ; n=6]$ in the medium-frequency range, with a mean improvement of $2.1 \mathrm{~dB}$. There was a significant effect of RW niche drilling $[\mathrm{F}(1,6)=6.5 ; \mathrm{p}=0.013$; $\mathrm{n}=6]$ in the high-frequency range with a mean difference of $4.22 \mathrm{~dB}$. The positive value indicates that drilling the RW niche produced a significant improvement in $H_{E V}$ of $4.22 \mathrm{~dB}$ with the $1.0-\mathrm{mm}$ ball tip. The value of $4.22 \mathrm{~dB}$ also exceeds the \pm 2.5 - $\mathrm{dB}$ variability assessed in the control experiments (subsection Control Experiment).

Figure $7 \mathrm{~b}$ shows the $\Delta H_{E V}$ for the 0.5 -mm ball tip with the RW niche intact and after drilling. There was no significant effect of drilling the RW niche $[\mathrm{F}(1,9)=0.37$; $\mathrm{p}=0.54 ; \mathrm{n}=6]$ in the low-frequency range, with a mean of $0.6 \mathrm{~dB}$. There was a significant effect of drilling the RW niche $[\mathrm{F}(1,8)=12.45 ; \mathrm{p}=0.007 ; \mathrm{n}=6]$ in the mid-frequency range, with a mean improvement of $5.6 \mathrm{~dB}$. There was also a significant effect $[\mathrm{F}(1,6)=17.4 ; \mathrm{p}=0.0001 ; \mathrm{n}=$ $6]$ in the high-frequency range, with a mean improvement of $10.2 \mathrm{~dB}$. The positive values of 5.6 and $10.2 \mathrm{~dB}$ in the mid- and high-frequency ranges, respectively, indicate that drilling the RW niche significantly improved performance with the $0.5-\mathrm{mm}$ ball tip. The values of 5.6 and $10.2 \mathrm{~dB}$ also exceeded the \pm 2.5 - $\mathrm{dB}$ variability assessed in the control experiments (subsection Control Experiment).

\section{Discussion}

Why Does Tip Size and Drilling the Lip of the RW

Niche Affect Performance?

First, from a surgical point of view, drilling the niche increases the surgical visualization of the RW membrane, and this fact makes the positioning of the AMEI on the RW more accurate. Second, particularly with the $1.0-\mathrm{mm}$ tip, the tip may come into contact with the bony niche in addition to the RW. The additional contact with the niche would be expected to reduce the direct transmission of vibratory energy to the RW, thus reducing performance. A positioning of the tip parallel to the visual access through the posterior tympanotomy occludes some part of the RW membrane, especially with an intact niche (without drilling) and with a $1.0-\mathrm{mm}$ tip. However, even in this case, because we took great care to consistently position the tip with the same loading parameters (accessed via the TLA data), within each temporal bone, we were confident that the position of the tip on the RW was approximately the same across all experimental manipulations. Although we cannot rule out the possibility that there was some minor contact of the 1-mm AMEI tip 
with the intact niche, we were confident that the tip was mostly in contact with the RW for two reasons. First, relative to the unloaded condition, the drops in impedance and inductance of the AMEI for a given distance of advance are vastly different when in contact with a hard surface like bone (or even the incus) compared to a more compliant surface like the RW. Second, when the AMEI is loaded purposefully on adjacent bone instead of the $\mathrm{RW}$, the performance as assessed by stapes velocity drops precipitously.

The lack of improvement at the low and mid frequencies might be explained by a remaining tip contact with the niche. According to Cervera-Paz et al. [2004], the surgical exposure of the RW membrane produces a mean diameter of $0.92 \mathrm{~mm}$. Clearly, a $1.0-\mathrm{mm}$ ball tip is of approximately the same diameter or larger than the distal end of the niche, and thus the tip is likely to be in contact with the rim of the membrane in addition to the RW. This latter hypothesis is difficult to assess since the inferior (proximal) part of the RW niche is hidden completely by the size of the ball (fig. 1b). For these reasons, the smaller diameter of the $0.5-\mathrm{mm}$ tip might be useful simply because it allows better visualization and a concomitant increased probability of having the tip in full contact with the RW membrane, even with the niche intact: using the $0.5-\mathrm{mm}$ tip, the increase in performance relative to the $1.0-\mathrm{mm}$ tip was significant both at medium and high frequencies, respectively. With the $0.5-\mathrm{mm}$ tip, the whole circumference of the membrane is visible and allows adequately placing the tip without any bone contact (fig. 1c).

\section{Clinical Importance of Present Study}

Drilling the niche certainly increases the ease of access to the RW membrane [Roland et al., 2007] and also makes the coupling of the tip to the RW more complete, as demonstrated objectively in this study. On the other hand, drilling directly on the bony part of the cochlea might induce an additional hearing loss (noise- or vibration-induced sensorineural [Pau et al., 2007]) and may also increase the risk of direct trauma to the RW membrane. Leaving the bony lip of the RW niche intact occludes the RW membrane and might increase the risk of RW rupture when fitting the tip into the niche. The $0.5-\mathrm{mm}$ tip along with RW niche drilling produced a mean advantage of $6 \mathrm{~dB}$ for all frequencies (ranging between $0.6 \mathrm{~dB}$ below $1 \mathrm{kHz}$ and $10.2 \mathrm{~dB}$ above $3 \mathrm{kHz}$ ). This benefit at high frequencies obtained by drilling the niche must be put into perspective with (1) the auditory thresholds of the patient and (2) the iatrogenic risks, as an overloading could damage the RW membrane, and drilling the niche may induce an additional sensorineural hearing loss.

\section{Rationale for RW Stimulation by AMEI}

Mechanical stimulation of the inner ear via the RW has long been known to be possible, and was demonstrated qualitatively in some of the classical studies of the cochlea [Wever and Lawrence, 1948]. Using an AMEI, Dumon et al. [1995] confirmed in an animal study the potential for RW stimulation by comparing measurements of acoustically evoked potentials to those evoked by piezoelectric vibrators implanted in the RW. Others have also confirmed this route of stimulation in animal-model studies of RW stimulation [Spindel et al., 1991, 1995; Zennaro et al., 1992; Koka et al., 2009] by both electromagnetic and piezoelectric AMEI technologies.

Despite the apparent and successful clinical outcomes in a limited number of patients [Colletti et al., 2006; Beltrame et al., 2009; Lefebvre et al., 2009; Martin et al., 2009; Tringali et al., 2009], few experimental data are available regarding the performance expectations of RW stimulation with an AMEI. In the present study, an AMEI was able to successfully drive the RW membrane in all cadaveric temporal bones with a classic facial recess approach. Cadaveric temporal bones are appropriate models for this type of performance assessment as previous reports [Chien et al., 2006, 2009] demonstrated similar acoustic stapes velocity transfer function measurements in both cadaveric temporal bones and live patients. In the normal ear, sound stimulation of the tympanic membrane by air conduction results in the motion of the stapes footplate with a corresponding motion in the cochlear fluid. This motion of the stapes footplate is also reflected in a comparable motion of the RW membrane, at least for low frequencies where the fluid volume displacement at the oval window nearly equals that at the RW, but with an opposite phase [Kringlebotn, 1995; Stenfelt et al., 2004a]. Thus, the RW membrane motion can be used to assess the sound transfer function through the cochlea when the oval window (or stapes) is not usable [Stenfelt et al., 2004b].

\section{Estimation of Maximum Equivalent Ear Canal Sound} Pressure Level Based on Stapes Velocity

There are published standards [Rosowski et al., 2007; ASTM, 2005] for the objective measurement of AMEI performance based on stapes velocity in human cadaveric temporal bones for AMEI when coupled to the ossicular chain. In these cases, stapes velocity is a reasonable measure of the input to the cochlea via the AMEI 
because the system is driven in the forward direction. However, these standards may not be applicable when estimating the efficiency of the RW drive with an AMEI because the stapes velocities in this case would be expected to be more complex due to differences in acoustic impedance between the middle ear and cochlea when driving the cochlea in the reverse direction, and due to the potential effects of 'third windows' [Stenfelt et al., 2004a; Puria, 2003]. The consequence of these factors would most likely lead to an underestimate of the performance of an AMEI when coupled to the RW.

With these limitations in mind, it is still useful to compute an estimate of the absolute performance, the maximum equivalent ear canal sound pressure level $\left(L_{\text {Emax }}\right)$, based on the standards above for ossicular stimulation with an AMEI in forward direction. Here, the $L_{E \max }$ based on stapes velocity in response to the AMEI stimulation of the RW was calculated by the equation given by Rosowski et al. [2007]:

$$
\begin{aligned}
L_{\text {Emax }} & =20 \cdot \log _{10}\left(P_{E \max } / 2 \cdot 10^{-5} \mathrm{~Pa}\right) \\
& =20 \cdot \log _{10}\left[\left(H_{E V} / H_{T V}\right) \cdot E_{\max } / 2 \cdot 10^{-5} \mathrm{~Pa}\right] .
\end{aligned}
$$

A maximum input voltage $\left(E_{\max }\right)$ of $1 \mathrm{~V}_{\text {rms }}$ to the AMEI (based on the manufacturer's instructions) was used along with the $H_{E V}$ and $H_{T V}$ for each of the 10 temporal bones. The mean $L_{E \max }$ measured across the 10 temporal bones with the RW niche intact was 95, 98 and $104 \mathrm{~dB}$ SPL for the 1-mm ball tip, and 95, 95 and $109 \mathrm{~dB}$ SPL for the $0.5-\mathrm{mm}$ ball tip, in the low-, medium- and high-frequency ranges, respectively. Given the limitations of the method to compute the $L_{E \max }$ mentioned above, we would expect the AMEI on the RW to produce at least this level of performance.

\section{Conclusion}

Mechanical stimulation of the RW membrane by an AMEI in cadaveric temporal bones was achieved using a classic and surgically relevant facial recess approach. Our findings demonstrate that a stimulation of the RW membrane by an AMEI without drilling the RW niche was sufficient for successful hearing outputs, particularly with a $0.5-\mathrm{mm}$ tip. However, using an AMEI tip of 0.5 $\mathrm{mm}$ diameter along with drilling the RW niche facilitates the optimal placement of the AMEI tip onto the RW, resulting in a significantly increased performance (5.6-10.2 $\mathrm{dB}$ ) for stimulus frequencies of $>1 \mathrm{kHz}$ relative to that with the niche intact.

\section{Acknowledgments}

We would like to acknowledge the technical and material support provided by Jim Easter and Brian Conn of Otologics LLC. We would like to thank Dr. Eric J. Lupo, Heath Jones and two anonymous reviewers for valuable comments and suggestions. We appreciate the assistance of Dr. Michael Hall for constructing custom experimental equipment (supported by NIH grant P30 NS041854-05). This study was supported in part by an Otologics LLC educational grant (H.A.J. and D.J.T.) and Department of Physiology and Biophysics funds (D.J.T.).

\section{References}

ASTM International F2504-05: Standard Practice for Describing System Output of Implantable Middle Ear Hearing Devices. Philadelphia, ASTM, 2005.

Barbara M, Manni V, Monini S: Totally implantable middle ear device for rehabilitation of sensorineural hearing loss: preliminary experience with the Esteem, Envoy. Acta Otolaryngol 2009;129:429-432.

- Beltrame AM, Martini A, Prosser S, Giarbini N, Streitberger C: Coupling the Vibrant Soundbridge to cochlea round window: auditory results in patients with mixed hearing loss. Otol Neurotol 2009;30:194-201.

-Cervera-Paz FJ, Linthicum FH, Manrique MS, Pérez N: Morphometry of the human cochlear wall and implications for cochlear surgery. Acta Otolaryngol 2004; 124:11241130 .

RW Stimulation
Chien W, Ravicz ME, Merchant SN, Rosowski JJ: Jenkins HA, Atkins JS, Horlbeck D, Hoffer ME, The effect of methodological differences in the measurement of stapes motion in live and cadaver ears. Audiol Neurootol 2006;11: 183-197. Balough B, Alexiades G, Garvis W: Otologics fully implantable hearing system: phase I trial 1-year results. Otol Neurotol 2008;29:534541.

Chien W, Rosowski JJ, Ravicz ME, Rauch SD, Jenkins HA, Atkins JS, Horlbeck D, Hoffer ME, Smullen J, Merchant SN: Measurements of Balough B, Arigo JV, Alexiades G, Garvis W: stapes velocity in live human ears. Hear Res US phase I preliminary results of use of the 2009;249:54-61.

Colletti V, Soli SD, Carner M, Colletti L: Treatment of mixed hearing losses via implantation of a vibratory transducer on the round window. Int J Audiol 2006;45:600-608.

Dumon T, Zennaro O, Aran JM, Bébéar JP: Piezoelectric middle ear implant preserving the ossicular chain. Otolaryngol Clin North Am 1995;28:173-187. Otologics MET Fully-Implantable Ossicular Stimulator. Otolaryngol Head Neck Surg 2007;137:206-212.

-Jenkins HA, Niparko JK, Slattery WH, Neely JG, Fredrickson JM: Otologics Middle Ear Transducer Ossicular Stimulator: performance results with varying degrees of sensorineural hearing loss. Acta Otolaryngol 2004;124:391-394.

Hüttenbrink KB, Zahnert T, Bornitz M, Beutner Kasic JF, Fredrickson JM: The Otologics MET D: TORP-vibroplasty: a new alternative for the chronically disabled middle ear. Otol Neurotol 2008;29:965-971. ossicular stimulator. Otolaryngol Clin North Am 2001;34:501-513. 
Koka K, Holland NJ, Lupo JE, Jenkins HA, Tollin DJ: Electrocochleographic and mechanical assessment of round window stimulation with an active middle ear prosthesis. Hear Res 2009; doi:10.1016/j.heares.2009.08.009.

Kringlebotn M: The equality of volume displacements in the inner ear windows. J Acoust Soc Am 1995;98:192-196.

- Lefebvre PP, Martin C, Dubreuil C, Decat M, Yazbeck A, Kasic J, Tringali S: A pilot study of the safety and performance of the Otologics fully implantable hearing device: transducing sounds via the round window membrane to the inner ear. Audiol Neurootol 2009; 14:172-180.

-Martin C, Devèze A, Richard C, Lefebvre PP, Decat M, Ibañez LG, Truy E, Mom T, Lavieille JP, Magnan J, Dubreuil C, Tringali S: European results with totally implantable carina placed on the round window: 2-year followup. Otol Neurotol 2009;30:1196-1203.

-Nadol JB Jr: Techniques for human temporal bone removal: information for the scientific community. Otolaryngol Head Neck Surg 1996;115:298-305.

- Pau HW, Just T, Bornitz M, Lasurashvilli N, Zahnert T: Noise exposure of the inner ear during drilling a cochleostomy for cochlear implantation. Laryngoscope 2007;117:535540.

Puria S: Measurements of human middle ear forward and reverse acoustics: implications for otoacoustic emissions. J Acoust Soc Am 2003;113:2773-2789.
Roland PS, Wright CG, Isaacson B: Cochlear implant electrode insertion: the round window revisited. Laryngoscope 2007;117:13971402.

-Rosowski JJ, Chien W, Ravicz ME, Merchant SN: Testing a method for quantifying the output of implantable middle ear hearing devices. Audiol Neurootol 2007;12:265-276.

Siegert R, Mattheis S, Kasic J: Fully implantable hearing aids in patients with congenital auricular atresia. Laryngoscope 2007;117:336340 .

Spindel JH, Corwin JT, Ruth RA, Lambert PR: The basis for a round window electromagnetic implantable hearing aid. Proc Ann Int Conf IEEE 1991;13:1891-1892.

Spindel JH, Lambert PR, Ruth RA: The round window electromagnetic implantable hearing aid approach. Otolaryngol Clin North Am 1995;28:189-205.

$\checkmark$ Stenfelt S, Hato N, Goode RL: Fluid volume displacement at the oval and round windows with air and bone conduction stimulation. J Acoust Soc Am 2004a;115:797-812.

-Stenfelt S, Hato N, Goode RL: Round window membrane motion with air conduction and bone conduction stimulation. Hear Res 2004b;198:10-24.
Sterkers O, Boucarra D, Labassi S, Bebear JP, Dubreuil C, Frachet B, Fraysse B, Lavieille JP, Magnan J, Martin C, Truy E, Uziel A, Vaneecloo FM: A middle ear implant, the Symphonix Vibrant Soundbridge: retrospective study of the first 125 patients implanted in France. Otol Neurotol 2003;24:427-436.

-Tringali S, Pergola N, Berger P, Dubreuil C: Fully implantable hearing device with transducer on the round window as a treatment of mixed hearing loss. Auris Nasus Larynx 2009;36:353-358.

Tringali S, Pergola N, Ferber-Viart C, Truy E, Berger P, Dubreuil C: Fully implantable hearing device as a new treatment of conductive hearing loss in Franceschetti syndrome. Int J Pediatr Otorhinolaryngol 2008;72:513517.

Venail F, Lavieille JP, Meller R, Devèze A, Tardivet L, Magnan J: New perspectives for middle ear implants: first results in otosclerosis with mixed hearing loss. Laryngoscope 2007; 117:552-555.

Wever EG, Lawrence M: The functions of the round window. Ann Otol Rhinol Laryngol 1948;57:579-589.

Willi UB, Ferrazzini MA, Huber AM: The incudo-malleolar joint and sound transmission losses. Hear Res 2002;174:32-44.

Zennaro O, Dumon T, Erre JP, Guillaume A, Aran JM: Piezo-electric middle ear implant hearing aid experimental model in guineapig. Proc Ann Int Conf IEEE 1992;4:13161317. 